\title{
Wireless Multi-hop Ad-hoc Networks: A Review
}

\author{
Chander Prabha ${ }^{1}$, Dr. Surender Kumar ${ }^{2}$, Dr. Ravinder Khanna ${ }^{3}$ \\ ${ }^{1}$ (Research Scholor, Department of Computer Science \& Engg., M.M.University, Sadopur, Ambala, India) \\ ${ }^{2}$ (Associate Professor, Department of Computer Science \& Engg. , HCTM, Kaithal, Ambala, India) \\ ${ }^{3}$ (Professor, Electronics \& Communication Engg. Department, M.M University, Sadopur, Ambala, India)
}

\begin{abstract}
Over the past decade wireless multi-hop ad-hoc networks have received a tremendous amount of research focus. Mobile devices equipped with wireless short range communication technologies make possible new applications for spontaneous communication, interaction and collaboration. The collaboration is used to facilitate communication when mobile devices are not able to establish direct communication paths. So the communication is multi-hop with intermediate nodes acting as routers that forward the messages addressed to other nodes. The concept of multi-hop ad-hoc networking was successfully applied in several classes of networks. This paper reviews the categories of wireless multi-hop ad-hoc networks and discusses main evolutions of wireless multi-hop ad-hoc networks specially the opportunistic networks.
\end{abstract}

Keywords: Delay tolerant Network, MANET, Mobile Devices, Opportunistic Network, WLAN

\section{Introduction}

In early days, most of the wireless networks were wireless local area networks (WLAN) operating the IEEE 802.11 WiFi protocol in infrastructure mode. The infrastructure consists of a base station, also called an access point that is owned by a company or a network operator. Such networks were centralized and the base station controls the access to the communication channel. The base station was also used to connect the WLAN to the company network or to the Internet. IEEE 802.11 offers a second mode, the ad-hoc mode. It is used now a days in many emergency application scenarios [1]. For example, military operations, relief and disaster recovery operations etc. In this mode, there is no base station, and devices within the same wireless LAN communicate directly (i.e., in a single hop).

An extension of wireless LAN operating in ad hoc mode are multi-hop ad hoc networks. They are typically deployed in large areas. A wireless multi-hop ad-hoc network is a network of nodes (e.g. computers, mobile nodes etc.) connected by wireless communication links. The links are usually implemented with digital packet radios. The transmission range of radio is very limited. Some devices might not be able to communicate directly to each other because of their limited radio range. These networks need other intermediate nodes to forward messages. In such cases, intermediary devices act as relays. In other words, the communication goes through multiple hops before reaching its final destination. This networking concept was successfully applied in several classes of networks that are penetrating the mass market [2]. These networks in various forms e.g. wireless sensor networks, vehicular networks, mesh networks and under various names e.g. ad-hoc networks, hybrid networks, delay tolerant networks and intermittently connected networks, are being increasingly used in military and civilian applications too. They are not relying on existing infrastructure hence their deployment cost is low. Also, they offer a potential throughput gain. These characteristics make multi-hop ad hoc networks a promising technology.

The remaining part of this paper is organised as follows. Section 2 gives a background of wireless multi-hop ad-hoc networks, as well as its application areas and various research issues are also discussed. Section 3 describes the main evolutions of wireless multi-hop ad-hoc networks specially the opportunistic networks. In section 4, routing challenges related to wireless multi-hop networks are discussed. Finally, section 5 concludes the paper and present the future work.

\section{Background}

The areas in which there is little or no communication infrastructure or the existing infrastructure is expensive or inconvenient to use, wireless mobile users may still be able to communicate through the formation of an ad hoc network. In such a network, each mobile node operates not only as a host but also as a router, forwarding packets for other mobile nodes in the network that may not be within direct wireless transmission range of each other. Every node that participates in an ad hoc routing protocol, allows it to discover "multi-hop" paths through the network to any other node. The idea of ad-hoc networking is sometimes also called infrastructure-less networking [3], since the mobile nodes in the network dynamically establish routing among themselves to form their own network. Some examples of the possible use of ad-hoc networking include students using laptop computers to participate in an interactive lecture, business associates sharing information 
during meeting, soldiers relaying information for situational awareness on the battlefield etc. [4]. Figure 1 shows the hierarchy/categories of wireless ad-hoc networks.

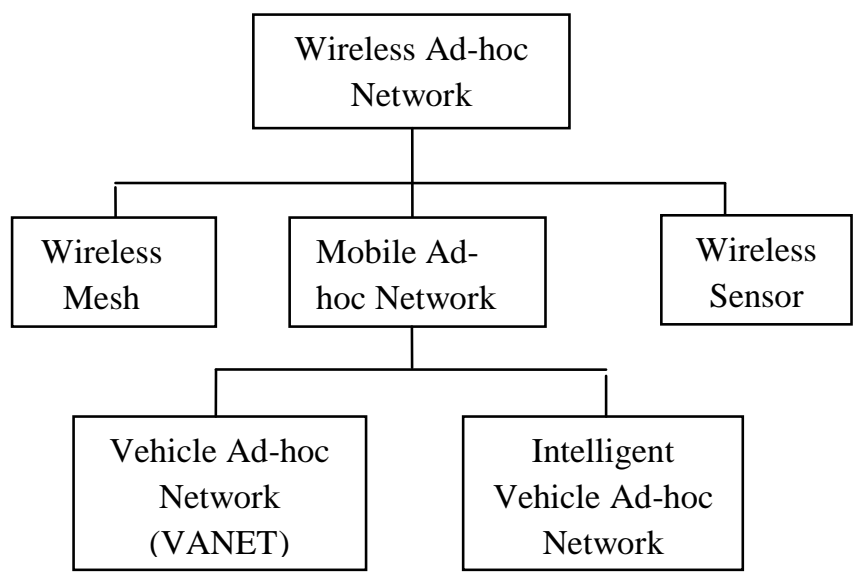

Fig. 1 Hierarchy/categories of Wireless Ad-hoc Networks

\subsection{Wireless Ad-hoc Network}

A wireless ad hoc network is a decentralized type of wireless network. The network is called ad-hoc because it does not rely on a pre-existing infrastructure, such as routers in wired networks or access points in managed (infrastructure) wireless networks. Instead, each node participates in routing by forwarding data for other nodes, so the determination of which nodes forward data is made dynamically on the basis of network connectivity [5]. It is a wireless multi-hop network where routing is done on hop by hop basis. Fig. 2 shows wireless ad-hoc network. It consist of devices that are wirelessly configured having their own transmission range.

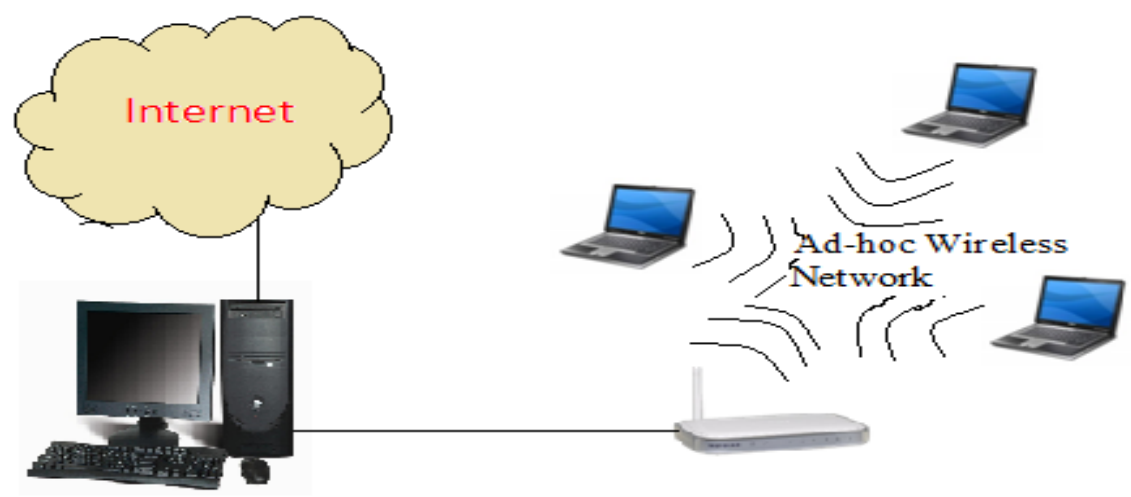

Fig. 2 Wireless Ad-hoc Network

Thus, an ad-hoc network refers to any set of networks where all devices have equal status on a network and are free to associate with any other ad- hoc network device in link range. Ad hoc network often refers to a mode of operation of IEEE 802.11 wireless networks [6]. The earliest wireless ad hoc networks were the "packet radio" (PRNETs) from the 1970s, sponsored by DARPA after the ALOHAnet project.

\section{Applications}

The decentralized nature of wireless ad hoc networks makes them suitable for a variety of applications where central nodes can't be relied on and may improve the scalability of networks compared to wireless managed networks. Minimal configuration and quick deployment make ad hoc networks suitable for emergency situations like natural disasters or military conflicts. The presence of dynamic and adaptive routing protocols enables ad hoc networks to be formed quickly [12].

\subsection{Mobile Ad-hoc Network}

A mobile ad-hoc network (MANET) is a set of mobile nodes which communicate over a multi-hop radio network, without relying on a stable infrastructure i.e. on infrastructure-less networks where nodes can 
move freely [7]. Multi-hop radio networks have arbitrary topology and packets need to be routed via hopping through a sequence of adjacent nodes. Since the nodes are moving, the network topology regularly changes. Obviously, exchange of information in multi-hop radio networks is more challenging than its counterpart i.e. in single-hop radio networks. In single hop radio network each pair of nodes can communicate directly. Fig. 3 shows mobile ad-hoc network. The mobile nodes are independent computing devices equipped with wireless transceivers.

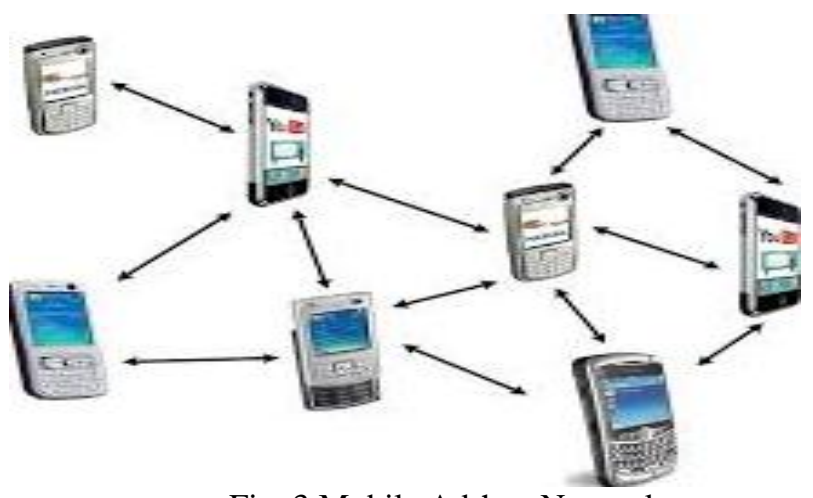

Fig. 3 Mobile Ad-hoc Network

These devices can exchange data with each other in a multi-hop fashion, where each of the nodes is able to act as a router to forward the packets. In these networks, nodes are usually battery-operated devices that can communicate via radio with other devices that are in range. Due to unreliable power supply and mobility, nodes may have a continuously changing set of neighbours in that range. The primary challenge in MANETs is continuously to maintain the routing information at each node required to properly route traffic.

\section{Applications}

The applications of MANETs include search-and-rescue operations. Such scenarios are characterized by a lack of installed communications infrastructure because all the equipment might already be destroyed or the region could be too remote. MANETs can also provide communications between autonomous vehicles, aircraft and ground troops in the battlefield where a fixed communication infrastructure is always unavailable and infeasible [4][8]. The applications of MANET are diverse ranging from emergency services, commercial environment, home and enterprise networking, educational environment etc.

\section{Research Issues}

Ad-hoc networking has been a popular field of study during the last few years. In MANET use of broadcasting and shared transmission media introduces a greater probability of packet collision and media contention. Irrespective of routing, variety of research issues are there including energy conservation, reliability, scalability, Quality of service, security, power control, node cooperation etc.

\subsection{Wireless Mesh Network}

A wireless mesh network (WMN) is a communication network made up of radio nodes organized in a mesh topology. These are another type of wireless multi-hop ad-hoc network, and are usually used to provide the last mile wireless broadband Internet access for the civilian users. They also support enterprise networking, healthcare and medical systems, and security surveillance systems. Fig. 4 shows wireless mesh network. Wireless mesh networks consist of mesh clients (nodes), mesh routers and gateways.

The mesh clients can be either stationary or mobile, and can form a client mesh (multi-hop) network among themselves and with mesh routers. The coverage area of the radio nodes working as a single network is sometimes called a mesh cloud. Access to this mesh cloud is dependent on the radio nodes working in harmony with each other to create a radio network. A mesh network is reliable and offers redundancy. When one node can no longer operate, the rest of the nodes can still communicate with each other, directly or through one or more intermediate nodes. Wireless mesh networks can be implemented with various wireless technology including 802.11, 802.15, 802.16, cellular technologies or combinations of more than one type. The integration of wireless mesh networks with other networks such as the Internet, cellular networks, IEEE 802.11 WLAN, IEEE 802.15, IEEE 802.16, and sensor networks can be accomplished through the gateway and bridging functions in the mesh routers. 


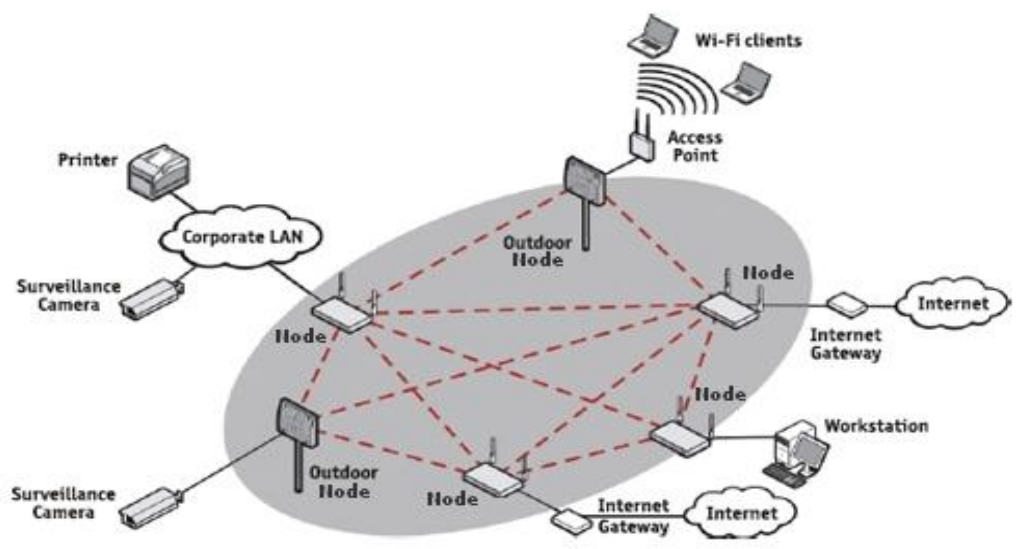

Fig. 4 Wireless Mesh Network

Network capacity in wireless mesh network is an important issue. The capacity of mesh network is affected by many factors such as network topology, node density, traffic patterns, number of radios/channels used for each node, transmission power level, carrier sensing threshold, node mobility, and environment (indoor/outdoor), etc. A clear understanding of the relationship between network capacity and the above factors provides a guideline for protocol development, architecture design, deployment and operation of the network [9].

\section{Applications}

An important possible application for wireless mesh networks is VoIP. By using a Quality of Service scheme, the wireless mesh may support local telephone calls to be routed through the mesh. Some current applications of wireless mesh networks are:

- U.S. military forces are now using wireless mesh networking to connect their computers, mainly ruggedized laptops, in field operations.

- Broadband internet access

- Electric meters now being deployed on residences transfer their readings from one to another and eventually to the central office for billing without the need for human meter readers or the need to connect the meters with cables.

- The laptops in the one laptop per child program use wireless mesh networking to enable students to exchange files and get on the Internet even though they lack wired or cell phone or other physical connections in their area.

\section{Research Issues}

The available MAC and routing protocols applied to wireless mesh networks do not have enough scalability as throughput drops significantly as the number of nodes or hops increases. Existing security schemes may be effective for certain types of attacks, but they lack a comprehensive mechanism to prevent attacks from different protocol layers. Similar problems exist in other networking protocols. Due to this, existing communication protocols, ranging from routing, MAC, and physical layers in the wireless mesh networking, need to be revisited and must be enhanced to ensure good routing performance as well as security problems[10][11].

\subsection{Wireless Sensor Networks}

Wireless sensor networks are another variant of multi-hop wireless network [12]. They are normally used to monitor various physical or environmental conditions, such as temperature, sound, vibration, pressure, motion or pollutants. A large-scale wireless sensor network typically consists of hundreds or thousands of small and cheap sensor nodes with wireless communication capabilities. These sensor nodes may form local clusters, and reactively or periodically report the sensing results to one or multiple base stations via multi-hop routing. Fig. 5 shows a wireless sensor network.

The sensors are usually powered by batteries with limited capacity. The information collected by the sensor nodes is delivered in a multi-hop fashion to a sink node and through this to the nodes connected to the internet. Energy efficiency is therefore the primary concern and key challenge in wireless sensor network. The sensors are typically static but some more powerful sensor nodes may have mobile capability. The aim of WSN is to collect information about events occurring in the sensor field, rather than to support the communication between nodes. 


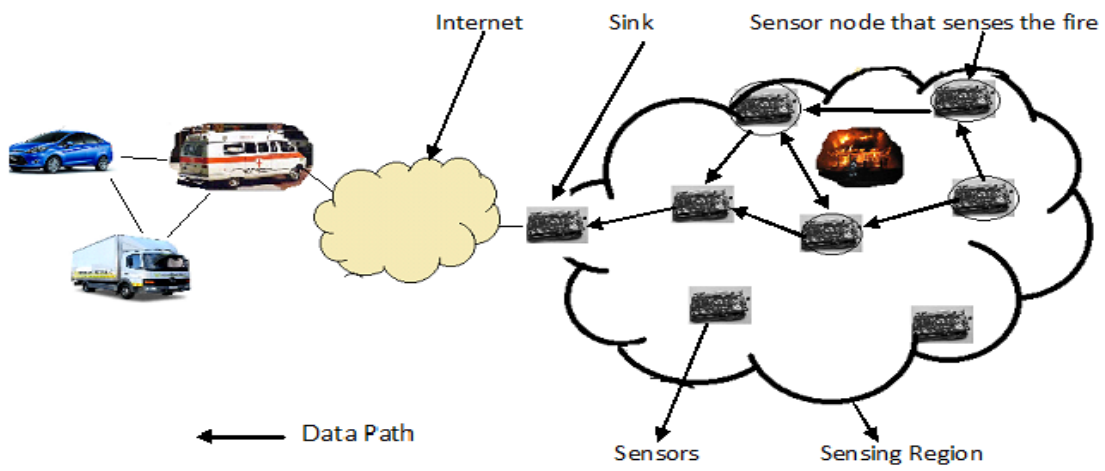

Fig. 5 A Wireless Sensor Network

\section{Applications}

Wireless sensor networks will not only enhance existing sensor network applications such as tracking, home automation, and environmental monitoring, health applications, but they will also enable several new applications such as multimedia surveillance sensor networks, advanced health care delivery, person locator services, industrial control and automation, security and public safety, agricultural monitoring(Sensors detect temperature, light levels and soil moisture at hundreds of points across a field and communicate their data over a multi-hop network for analysis)., iButton [13] etc.

\section{Research Issues}

There is need to developed new protocols to address higher topology changes and higher scalability issues as well as to allow easy communication between the sensor networks and external networks.

\subsection{VANETs and InVANETs}

In VANETs, every vehicle communicates with other vehicles (V2V) and with roadside infrastructures (V2I) by means of wireless communication equipment. VANETs are a cornerstone of the envisioned Intelligent Transportation Systems (ITS). They will contribute to safer and more efficient roads in the future by providing timely information to drivers and concerned authorities [14]. VANETs are similar to MANETs, but the key difference lies in that in VANETs, vehicles move in an organized fashion rather than randomly. The vehicles are restricted in their range of motion and their mobility can be predicted in the short term, because their movement should obey certain traffic rules.

In 2006 the term MANET mostly described an academic area of research, and the term VANET an application. Such a network might pose safety concerns (for example, one cannot safely type an email while driving). GPS and navigation systems might benefit, ${ }^{[2]}$ as they could be integrated with traffic reports to provide the fastest route to work. It was also promoted for free, VoIP services such as Google Talk or Skype between employees, lowering telecommunications costs.

Intelligent vehicular ad hoc networks (InVANETs) use WiFi IEEE 802.11p (WAVE standard) and WiMAX IEEE 802.16 for easy and effective communication between vehicles with dynamic mobility. Effective measures such as media communication between vehicles can be enabled as well methods to track automotive vehicles. InVANET is not foreseen to replace current mobile(cellular phone) communication standards . Within the IEEE Communication Society, there is a Technical Subcommittee on Vehicular Networks \& Telematics Applications (VNTA). The charter of this committee is to actively promote technical activities in the field of vehicular networks, V2V, V2R and V2I communications, standards, communications-enabled road and vehicle safety, real-time traffic monitoring, intersection management technologies, future telematics applications, and ITS-based services [15].

\section{Applications}

The most important usage of these networks is to inform other vehicles in emergency situations such as car accidents, urgent braking or traffic jams. In such cases, a vehicle can inform other vehicles by broadcasting safety messages before facing the event. Applications of VANET are mainly oriented towards safety issues for example traffic services, alarm and warning messaging. Other applications such as traffic and congestion monitoring require collecting information from vehicles that span multiple kilometers. Non-safety applications are expected to create new commercial opportunities by increasing market penetration of the technology and making it more cost effective. 


\section{Research Issues}

Currently there is ongoing a lot of research in the field of InVANETs for several scenarios. The main interest is in applications for traffic scenarios, mobile phone systems, sensor networks and future combat systems. Recent research has focused on topology related problems such as range optimization, routing mechanisms, or address systems, as well as security issues like traceability or encryption. In addition, there are very specific research interests such as the effects of directional antennas for InVANETs and minimal power consumption for sensor networks. Most of this research aims either at a general approach to wireless networks in a broad setting or focus on an extremely specific issue. From the very beginning, the VANET research area received strong support from the government (e.g. U.S. Department of Transportation), as well as from industry.

\section{Evolutions of Multi-hop Ad-hoc Networks}

There is a slow progress the development and deployment of commercial ad-hoc applications. This is due to the fact that original ad-hoc applications scenarios were not directed to mass users. An important new concept has emerged which may help extent ad-hoc networking to commercial applications, namely the concept of opportunistic ad-hoc networking.

There are two main evolutions of multi-hop Ad-hoc networks. One is mesh networks and second one is opportunistic networks. Opportunistic networks emerged as one of the interesting evolution of multi-hop ad-hoc networks. Current scenario in networking-architecture developments, like delay and disruption tolerant networks, and opportunistic networking, aim to deal with the disconnections that naturally and frequently arise in wireless environments [16,17]. Their objective is to allow communication in dynamic networks, like in a MANET, even if a direct route between sender and receiver does not exist in the network. Due to this fact, multi-hop communication is provided through opportunistic links, in which the route of a message is followed one link at a time, as other links in the route become available. When the next link is not available, the message is held in a node. With opportunistic communication, strong connectivity requirements are no longer needed. Furthermore, in some cases mobility is the key to allow communication (e.g. consider two disconnected static nodes, where communication between them is provided by a device that, due to mobility, sometimes is in range of one and sometimes of the other).

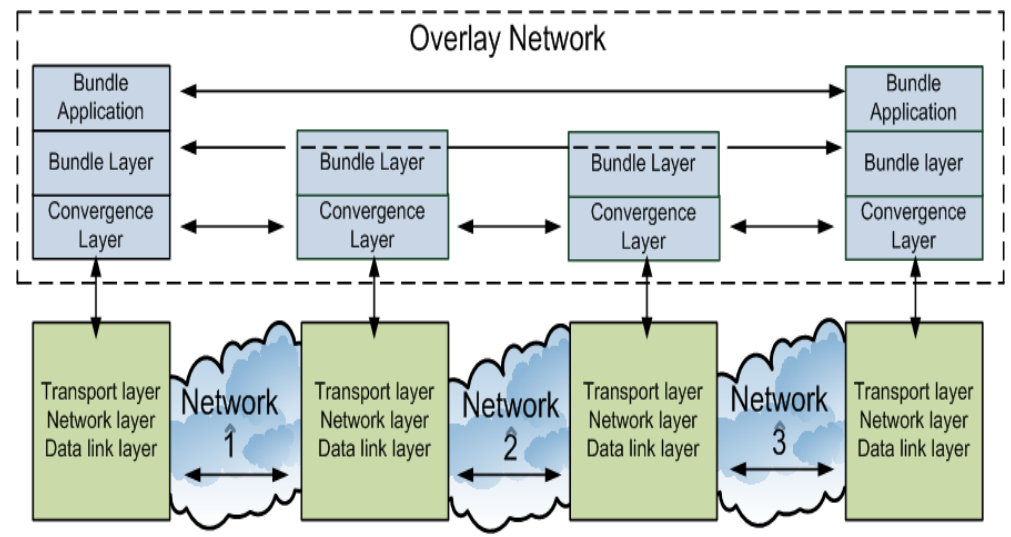

Fig. 6 DTN Architecture (From DTNRG)

In some scenarios [18] complete end-to-end paths rarely or never exist between sources and destinations within the MANET, due to high node mobility. These networks may experience frequent partitioning, with the disconnections lasting for long periods. As a consequence, the end-to-end transfer delays in these intermittently connected networks (ICNs) are much greater than typical IP data transfer delays in conventional networks such as the Internet. In the literature, intermittently-connected networks are often referred to as delay or disruption tolerant networks (DTN) [19]. Fig. 6 shows the architecture for delay tolerant network. It was developed by the Internet Research Task Force (IRTF) DTN Research Group (DTNRG) [20].

This architecture considers intermittently connected networks that suffer from frequent partitions. The opportunistic networks are an important class of DTNs [21] in which contacts appear opportunistically without any prior information. They cover many similar aspects of delay tolerant networks, which can be characterised by intermittent connectivity, frequent end-to-end path disruption and topology changes. Fig. 7, shows an example of DTN [17] connecting the ad-hoc network among the soldiers on a battlefield to the LAN on the nearest aircraft carrier. A helicopter is in charge of providing periodic connection between these two internets. Many of the concepts behind opportunistic networks comes from the studies of delay tolerant networks. In opportunistic network it is not mandatory to have a priori knowledge about the network topology whereas, DTN 
assumes the knowledge of Internet-like topologies in which some links between gateways could be available just at certain(possibly unspecified) times[22].



Fig. 7 Example of Delay Tolerant Network.

Routes in DTNs are typically computed via legacy-Internet techniques by taking into consideration the link unavailability. But in opportunistic networks, routes are computed at each hop while a packet is forwarded. So, each node receiving a message for an eventual destination exploits local knowledge of a node to decide which is the best next hop, among its current neighbours, to reach the final packet destination. When no forwarding opportunity exists (e.g., no other nodes are in the transmission range, or the neighbours that are evaluated are not suitable for that communication) the node stores the message and waits for future contact opportunities with other devices to forward the information.

However, in opportunistic networks each single node acts as a gateway. This makes opportunistic networks a more flexible environment than DTNs. For example (Fig. 8), the woman at the desktop opportunistically transfers, via a Wi-Fi link to his colleague seated at the other office. First the message reach to the wireless hub then after it moves to boy seated on ground with his laptop accessing the same wireless hub. Then this message is send to the men with his cars Bluetooth radio will carry the information closer to the destination. The men with car moves through the long distance, then uses its Bluetooth radio to forward the message to the final destination device while moving near by the office of the second men. As it is clearly shown in this example, a network connection between the two women never exists but, by opportunistically exploiting contacts among heterogeneous devices, the message is delivered hop-by-hop (hopefully) closer to the destination, and eventually to the destination itself. Besides allowing nodes that are not connected at the same time to the same network to communicate with each other, opportunistic networks are also a possible way to improve the capacity of multi-hop ad hoc networks [23].

Opportunistic networking tries to simplify the aspect of ubiquitous computing by removing the assumption of physical end-to-end connectivity while providing connectivity opportunities to pervasive devices when no direct access to the Internet is available. Ubiquitous computing is an advanced computing concept where computing is made to appear everywhere and anywhere. Pervasive devices (e.g. mobile devices, laptop, Bluetooth etc.) equipped with different wireless networking technologies, are frequently out of range from a network but are in the range of other networked devices, and sometimes cross areas where some type of connectivity is available (e.g. Wi-Fi hotspots). Thus, they can opportunistically exploit their mobility and contacts for data delivery $[3,17]$.

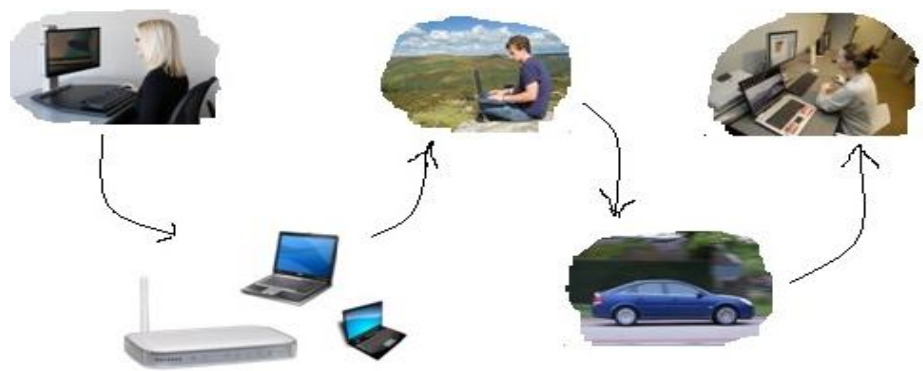

Fig. 8 Example of Opportunistic Networking 
In Opportunistic networking, sometimes also called disconnected MANET, no assumption is made on the existence of a complete path between two nodes wishing to communicate [23]. Source and destination node might never be connected to the same network. In these networks, communication devices can be carried by people, vehicles or animals, etc. Some devices can form a small mobile ad-hoc network when the nodes move close to each other. But a node may frequently be isolated from other nodes. Therefore, a node is just intermittently connected to other nodes, and this partitioning is dynamically changing with time. Thus, an endto-end connection between the source and the destination can be absent at the time the source wants to transmit, and even later.

\section{Applications}

Opportunistic networks can provide intermittent Internet connectivity to rural and developing areas where conventional networks do not exists and they only represent the affordable way to help bridge the gap between these two areas. DakNet and SNC (Sammi Network Connectivity) are good examples of the approach used by opportunistic networks. Another interesting application of opportunistic networks is wildlife monitoring by tracking wild species to deeply investigate their behaviour and understand the interactions and influences on each other, as well as their reaction to the ecosystem changes caused by human activities [24].

\section{Research Issues}

Disconnection of nodes and high churn rates are thus normal features of opportunistic networks. This sporadic connectivity of nodes is not only the challenge in opportunistic networks. Also routing, information dissemination, buffer capacities, jamming are some of the main challenges in disconnected environment of these networks. These challenges can limit the contribution of nodes in data forwarding, which in turn, effect the performance of network. So efficient routing protocols must be there which can manage the forwarding of data in disconnected environment.

\section{Routing Challenges}

The communication networks have become an integral part of our society to meet the needs of fast and reliable information exchange. Wireless ad- hoc networks will enhance communication capability significantly by providing connectivity from anywhere at any time. In any of the network, the purpose of routing is generally to find a path or multiple paths from the source to the destination, maintain or update path(s) when the topology or link quality changes, and forward packets along the path(s).

Routing protocol design in wireless multi-hop ad-hoc networks faces a great challenge mainly due to the facts that wireless link is unreliable, wireless medium is broadcast in nature, mobility, battery power constraints etc. These are some of the factors behind the research on routing protocols in wireless multi-hop adhoc networks. There are lot of protocols in the literature for wireless multi-hop ad-hoc networks to cope with different problems and meet different application requirement. These protocols are divided into different categories using different criteria.

Opportunistic Networks: Routing scheme in multi-hop ad-hoc network i.e. in opportunistic networks has to provide data with some reliability even when the network connectivity is intermittent or when an end-toend path is temporally non-existent[44]. Routing algorithms have to cope with the typical limitations of wireless networks: high power consumption, low wireless bandwidth, high error rates. In case of opportunistic networks, the design of efficient routing strategies is generally a complicated task due to the absence of knowledge about the topological evolution of network. Routing performance improves when more knowledge about the topology of the network can be exploited. But this kind of knowledge is not easily available in opportunistic networks due to unpredictable topology.

Routing performance improves when more knowledge about the expected topology of the network can be exploited. But in opportunistic network, this kind of knowledge is not easily available. The trade-off must be met between performance and knowledge requirement.

\section{Conclusion}

In this paper, we have reviewed and discussed various categories of wireless multi-hop ad-hoc networks, their application and research issues. In the early stages, the ad-hoc networks were expected to be used for specific applications by a limited set of users. The involvement of end users are the key elements of mesh, vehicular, sensor and opportunistic networks. There are a number of applications of these types of networks, ranging from small, static networks that are constrained by power sources to large-scale mobile, highly dynamic networks. We discuss the applicability and fundamental underlying ideas of opportunistic networks i.e. one of the main evolutions of wireless multi-hop ad-hoc networks. Research challenges specific to opportunistic networks are discussed and it requires a lot of research for improving the routing performance in 
these networks. The requirements of the opportunistic networks makes these networks a promising and challenging research field.

\section{References}

[1] M.Grossglauser and D.N.C.Tse, Mobility increases the capacity of Ad-hoc wireless network, IEEE/ACM Transactions on Networking, Vol. 10, no.4 Aug. 2002.

[2] P.Gupta and R.P Kumar, The Capacity of Wireless Networks, IEEE Transactions on Information Theory, Vol. 46, Mar. 2000, pp. 388-404.

[3] Macro Conti, Silvia Giordano, Multi-hop Ad-hoc networking: The Theory, IEEE Communications Magazine, pp.78-86, April 2007.

[4] Macro Conti, Silvia Giordano, Multi-hop Ad-hoc networking: The Reality, IEEE Communications Magazine, pp. 88-95, April 2007.

[5] IETF Mobile Ad-hoc Networking Group. http://www.ietf.org/html.charters/manet-charter.html.

[6] Mohammad Illyas, Handbook of Ad-hoc Wireless Network (CRC Press 2003).

[7] S.sarkar, T.G.Basavaraju, C.Puttamadappa, Adhoc Mobile Wireless Networks: Principles, protocols \& Applications, (Auerbach Publications, 2008)

[8] I.Chlamtac et al. Mobile Adhoc Networking: Imperatives \& Challenges, Adhoc Networks: Elsevier.

[9] I.F. Akyildiz \& X.Wang, A survey on wireless mesh networks, IEEE Communications Magazine, pp. 23-30, Nov. 2010.

[10] Mihail L.Sichitiu, Wireless Mesh Networks: Opportunities and Challenges, IEEE Communications Mazagine, pp. 73-80, Nov. 2011

[11] S.Seth et al. Current state of art research issues \& challenges in wireless mesh networks, Published in Computer Engg. \& Applications (ICCEA), $2^{\text {ND }}$ Intl. Conference, pp. 199-203, March 2010.

[12] I.F.Akyildiz \& I.H.Kasimoglu, Wireless sensor and actor networks: Research Challenges, Adhoc Network Journal(Elsevier), pp. 351-367, Dec. 2009.

[13] Z. J. Haas et al., Special Issue on Wireless ad hoc networks, IEEE Journal on Selected Areas in Communications, 17(8), Aug. 2009.

[14] Kamini, R.Kumar, VANET parameters and applications: A Review, Global Journal of Computer Science and Technology, Sept. 2010 .

[15] A.K.Thangavelu, S.Kannan, A middleware architectural framework for vehicular safety over VANET(InVANET), IEEE Explorer, NetCom Dec 2009.

[16] Julien Haillot, Frederic Guidec, Toward a use-net like discussion system for users of Disconnected MANETs, IEEE Computer Society, pp. 1678-1683, 2008.

[17] C.M.Huang, Kun-Chan \& Chang-Zhou Tsai, A survey of Opportunistic networks, IEEE Computer Society, pp. 1672-1677, 2008.

[18] Z.Zhang, Routing in intermittently connected mobile ad hoc network \& delay tolerant networks: Overview \& challenges, IEEE Communication Surveys \& Tutorials, Vol.8, No.1, pp. 24-37, 2006.

[19] F.Warthman, Delay tolerant network (DTNs): A Tutorial, DTNRG doc ver.1.1 3/5/03, 2003.

[20] Delay tolerant networking research group, http://www.dtrg.org.

[21] M.J.Khabbaz, C.M.Assi, W.F.Fawaj, Disruption tolerant networking: A comprehensive survey on recent developments \& persisting challenges, IEEE Communication surveys \& tutorials, vol.14, no.2, pp.607-640, 2012.

[22] Luciana Pelusi, Andrea Passarella, et.al, Data forwarding in disconnected mobile ad hoc networks, IEEE communication magazine, pp. 134-141, Nov, 2006.

[23] Yun Li, Jihong Yu. Et.al, A Novel Bargaining based Incentive Protocol for Opportunistic Networks, pp. 5285-5289, Globecom 2012: Wireless networking symposium, IEEE 2012

[24] Hua Zhu, Kejie Lu, Resilient Opportunistic Forwarding: Issues and Challenges, IEEE Computer Society, pp. 1543-1551, 2011. 